Tropical Journal of Pharmaceutical Research July 2020; 19 (7): 1465-1471

ISSN: $1596-5996$ (print); 1596-9827 (electronic)

(C) Pharmacotherapy Group, Faculty of Pharmacy, University of Benin, Benin City, 300001 Nigeria.

Available online at http://www.tjpr.org

Original Research Article

http://dx.doi.org/10.4314/tjpr.v19i7.19

\title{
Protective effect of erdosteine against methotrexate- induced hepatotoxicity in rats
}

\author{
Osama Abdelaziz Hassan ${ }^{1}$, Entesar Farghally Amin², Rabab Ahmed Moussa ${ }^{3 *}$ \\ ${ }^{1}$ Forensic Medicine and Clinical Toxicology Department, ${ }^{2}$ Pharmacology Department, ${ }^{3}$ Pathology Department, Faculty of \\ Medicine, Minia University, 61111, Minia, Egypt
}

*For correspondence: Email: rababmossa00@gmail.com; Tel: 00201004740750

Sent for review: 7 December 2019

Revised accepted: 23 June 2020

\begin{abstract}
Purpose: To study the possible mitigating effect of erdosteine (ERD) against methotrexate (MTX)induced liver toxicity.

Methods: Male albino Sprague-Dawley rats were randomly assigned to four groups of 8 rats each, viz, vehicle control, MTX (20 mg/kg i.p.), MTX (20 mg/kg i.p.) + ERD (300 mg/kg) and ERD (300 mg/kg) groups. Serum levels of alanine aminotransferase (ALT), and aspartate aminotransferase (AST) were determined by enzymatic colorimetric commercial kits while Hepatic tissue content of malondialdehyde $(M D A)$, reduced glutathione (GSH), SOD and catalase (CAT) were also evaluated. In addition, measurement of the inflammatory cytokine, TNF- $\alpha$, as well as histopathological examination and histochemical assessment were carried out.

Results: The results indicate that, compared to the control group, MTX group showed a remarkable elevation in oxidative stress as indicated by significantly lower levels of SOD, CAT and reduced glutathione, and increased tissue malondialdehyde $(p<0.05)$. MTX group exhibited significantly higher blood activities of ALT, AST and TNF- $\alpha$, reflective of hepatocyte damage and inflammation $(p<0.05)$. In MTX group, significant hepatic degenerative changes were detected on histological examination, while marked apoptotic alternations were observed following immunohistochemical analysis of caspase-3 expression, when compared to control group. However, administration of ERD to rats ameliorated the changes in these parameters $(p<0.05)$.

Conclusion: Treatment with ERD in rats produced alleviation in hepatic oxidative stress, apoptosis, inflammation, and histological damage, when compared to MTX group. This study is the first to demonstrate the potentially protective effect of ERD-pretreatment against hepatotoxicity associated with MTX.
\end{abstract}

Keywords: Erdosteine, Methotrexate, Hepatotoxicity, Oxidant, Anti-oxidant

\begin{abstract}
This is an Open Access article that uses a fund-ing model which does not charge readers or their institutions for access and distributed under the terms of the Creative Commons Attribution License (http://creativecommons.org/licenses/by/4.0) and the Budapest Open Access Initiative (http://www.budapestopenaccessinitiative.org/read), which permit unrestricted use, distribution, and reproduction in any medium, provided the original work is properly credited.

Tropical Journal of Pharmaceutical Research is indexed by Science Citation Index (SciSearch), Scopus, International Pharmaceutical Abstract, Chemical Abstracts, Embase, Index Copernicus, EBSCO, African Index Medicus, JournalSeek, Journal Citation Reports/Science Edition, Directory of Open Access Journals (DOAJ), African Journal Online, Bioline International, Open-J-Gate and Pharmacy Abstracts
\end{abstract}

\section{INTRODUCTION}

Methotrexate (MTX) is an antineoplastic and immunosuppressive drug that is frequently used for the treatment of multiple types of leukemia and solid tumors [1], as well as rheumatoid arthritis and psoriasis [2]. The antineoplastic action of MTX is mediated by its ability to inhibit nucleic acid and protein synthesis, thereby inhibiting tumor cell growth and division. It 
interferes with folic acid metabolism leading to inhibition of the synthesis of thymidine, purine and pyrimidine nucleosides required for RNA and DNA synthesis [3]. However, the folic acid pathway is not thought to underlie the immunosuppressive effect of MTX, which is rather attributed to the inhibition of $T$ and $B$ cell activation, blockage of the binding of $\|-1 \beta$ cytokine to its cell surface receptors, and inhibition of methyltransferase activity relevant to the immune system function [4]. Despite the wide use of MTX, which is currently on the WHO list of essential medicines, several guidelines recommend monitoring of hepatic function before and/or during long-term MTX use [5]. At high doses, MTX causes acute serum elevation of liver enzymes. In addition, prolonged use of MTX is known to cause persistent elevation of liver enzymes in blood, and more importantly, it has been associated with the development of chronic hepatic diseases such as progressive fibrosis, cirrhosis and portal hypertension [6].

The mechanism of hepatotoxicity with MTX is believed to involve direct toxicity. In addition to the inhibitory effect on DNA and protein synthesis, the hepatotoxic effect of MTX significantly involves reductions in methionine synthesis and various enzyme antioxidants e.g. CAT, GPx and SOD. Reductions in the activities of these enzymes result in build-up of ROS, leading to deleterious effects on DNA, proteins, lipids and various cell organelles, and enhancing apoptosis [7].

Erdosteine (ERD) is a mucolytic drug that is currently used for the treatment of chronic pulmonary diseases so as to enhance tracheobronchial clearance [8]. Recently, a significant body of evidence has highlighted the antioxidant effects of ERD, in addition to its established mucolytic affect [9]. It has been shown that erdosteine ameliorated oxidative tissue damage in various animal models of druginduced organ toxicity [10]. Based on these findings, it was felt that the potential mitigating influence of ERD pre-treatment against MTXmediated liver damage should be studied in a rat model of the disease. This study was done to determine the influence of MTX administration on various biochemical and pathological indicators of hepatic toxicity with or without the concomitant treatment with ERD.

\section{EXPERIMENTAL}

\section{Animals}

Adult male Sprague-Dawley rats weighing 200 $250 \mathrm{~g}$ were obtained from the Experimental
Animal Unit of Minia University. They were maintained under $12 \mathrm{~h}$ light:12 $\mathrm{h}$ dark photoperiod, cycle and were given standard laboratory diet and water ad libitum.

Animal handling and experimental protocols used were performed according to guidelines of US National Institutes of Health. The procedures used received approval from Ethics Board Committee of the Faculty of Medicine, Minia University.

\section{Study design and procedures}

The rats were randomly assigned to four groups (8 per group) as follows: vehicle control group (rats given $1 \mathrm{ml}$ of $0.5 \%$ carboxymethylcellulose daily orally for 7 days); MTX group (rats that received one i.p dose of MTX i.e. $20 \mathrm{mg} / \mathrm{kg}$ on day 3; MTX + ERD group [rats given ERD at a (300 $\mathrm{mg} / \mathrm{kg})$, orally, once a day continuously for 7 days, and one dose of MTX (20 mg/kg, i.p.) on the $3^{\text {rd }}$ day], and ERD group where rats received ERD (300 mg/kg orally) daily for 7 days [14,15].

On day 7 , the animals were subjected to $12 \mathrm{~h}$ fast, and blood was drawn from the neck vein of each rat under urethane anesthesia $(1.5 \mathrm{~g} / \mathrm{kg}$, i.p.), and sera samples were obtained after centrifugation at $5000 \mathrm{rpm}$ for $10 \mathrm{~min}$. The samples were frozen at $-80{ }^{\circ} \mathrm{C}$ for further analysis. The rat livers were excised. One portion of each liver sample was kept in $10 \%$ phosphate-buffered formaldehyde for histopathological and immunohistochemical analyses, while the other part was frozen in liquid nitrogen before homogenization in cold potassium phosphate buffer $(\mathrm{pH}$ 7.4) for further biochemical analyses.

\section{Assay of serum liver enzymes}

Serum ALT and AST were assayed colorimetrically in line with the procedure of Reitman and Frankel, using commercial kits (Spectrum Diagnostics, Cairo, Egypt) [11].

\section{Measurement of tissue oxidation indicators}

Hepatic tissue content of MDA was estimated with a spectrophotometric kit (Spectrum Diagnostics, Cairo, Egypt), based on the reaction of MDA with thiobarbituric acid as previously described [12]. Reduced glutathione (GSH) was estimated with a commercial kit (Spectrum Diagnostics, Cairo, Egypt), based on Ellman's spectrophotometric method [13]. Superoxide dismutase (SOD) was measured according to the method of Marklund [14]. Catalase (CAT) activity 
was assayed using a commercial colorimetric kit (Spectrum Diagnostics, Cairo, Egypt) [15].

\section{Measurement of the inflammatory cytokine, TNF- $\alpha$}

Serum levels of TNF- $\alpha$ were assayed with a commercial ELISA kit (ID Labs Inc, Ontario, Canada). The method is based on a solid phase sandwich ELISA utilizing a monoclonal capture antibody specific for rat TNFa coated on a 96well plate. The assay was performed according to the manufacturer's instructions, and the results were read spectrophotometrically at $450 \mathrm{~nm}$.

\section{Histopathological examination}

The liver portions were subjected to routine processing for light microscopy and subjected to standard $H$ \& $E$ stain, prior to microscopic examination. Pathological assessment of hepatic injury was performed for each experimental group based on lesions of nuclear pyknosis, cytoplasmic vacuolization, necrotic or inflammatory foci, and loss of architecture.

\section{Immunohistochemical staining for caspase-3}

This was done using streptavidin-biotin immuneperoxidase complex procedure. Adhesive slides were produced from $5 \mu \mathrm{m}$-thick sections of processed and paraffinized liver blocks. Xylene was used to remove paraffin from the liver sections, followed by dehydration in alcohol gradient and inhibition of intrinsic peroxidase by treatment with $0.3 \% \mathrm{H}_{2} \mathrm{O}_{2}$. Then, the sections were microwaved in citrate buffer, $\mathrm{pH} \mathrm{6}$, for 20 min, for cleaved caspase-3, followed by incubation with cleaved caspase-3 antibody. Rabbit polyclonal antibody (ThermoFisher Scientific, IL, USA) diluted 1:200, was applied, prior to biotinylated $2^{0}$ antibody for $0.5 \mathrm{~h}$ at room temperature. The reaction was visualized using an avidin-biotin complex immune-peroxidase method, with 3,3'diaminobenzidine as color developer. This was followed with counterstaining using $\mathrm{H}$ \& $\mathrm{E}$, dehydration, clearing, and mounting. Nuclear staining was considered positive for cleaved caspase-3. Assessment of immunostaining was performed via enumeration of positively-stained cells in 5 high-power fields at magnification of $x 400$.

\section{Statistical analysis}

Data are expressed as mean \pm SEM, and were statistically analyzed using SPSS, version 24 software. Statistical comparison was performed with one-way ANOVA test, followed by Tukey's post-hoc test. Differences were considered significant at $p<0.05$.

\section{RESULTS}

\section{ERD reduced MTX-induced elevation of serum liver enzymes}

The MTX group showed significant elevations in serum ALT and AST levels, relative to control and erdostiene-treated groups $(p<0.05$; Table 1). However, MTX + ERD treatment resulted in significant decreases in serum AST and ALT, when compared to the MTX group $(p<0.05)$. No significant differences in AST and ALT levels were seen between the control and ERD rats.

\section{ERD reduced MTX-induced hepatic oxidative stress and inflammation}

Rats in the MTX group had significant decreases in hepatic levels of the antioxidants GSH, CAT, and SOD, while there was marked increase in tissue concentration of the oxidative stress marker MDA, and reduced serum levels of the inflammatory indicator TNF- $\alpha$, relative to control and erdostiene-treated rats $(p<0.05$; Table 2). These effects were reversed in the MTX + ERD group by concomitant ERD treatment, resulting in significant changes in the levels of these parameters, when compared to the MTX group $(p<0.05)$. No significant differences in $\mathrm{GSH}$, CAT, SOD, MDA or TNF- $\alpha$ levels were observed between the control group and the group that received only ERD.

Table 1: Influence of ERD on biochemical indices in MTX-mediated hepatotoxicity

\begin{tabular}{lcccc}
\hline \multirow{2}{*}{ Parameter } & MTX+ERD & MTX & ERD & Control \\
\cline { 2 - 5 } & $33.34 \pm 2.47^{\#}$ & $48.35 \pm 2.14^{* @}$ & $31.65 \pm 1.99$ & $30.33 \pm 2.39$ \\
ALT(U/L) & $40 \pm 3.1^{\#}$ & $69.2 \pm 5.34^{*} @$ & $37.7 \pm 3.21$ & $37.3 \pm 2.52$ \\
AST(U/L) & & &
\end{tabular}


Table 2: Effect of ERD on oxidant/antioxidant and pro-inflammatory parameters in MTX-induced hepatotoxicity

\begin{tabular}{lcccc}
\hline \multirow{2}{*}{ Parameter } & \multicolumn{4}{c}{ Group } \\
\cline { 2 - 5 } & Control & ERD & MTX & MTX+ERD \\
\hline MDA (nmol/g tissue) & $44.92 \pm 1.83$ & $43 \pm 3.1$ & $80.83 \pm 4.57^{* @}$ & $53 \pm 2.28^{\#}$ \\
GSH (mg/g tissue) & $211.8 \pm 14.58$ & $210.9 \pm 14.44$ & $148.5 \pm 11.12^{* @}$ & $184 \pm 12.11^{\star @ \#}$ \\
SOD (U/g tissue) & $1055 \pm 70.52$ & $1057 \pm 87.36$ & $783.3 \pm 55.17^{\star @}$ & $1060 \pm 10.75^{\#}$ \\
CAT (U/g tissue) & $36 \pm 1.36$ & $34.9 \pm 2.4$ & $13.33 \pm 1.25^{\star} @$ & $28.10 \pm 1.59^{\star} @ \#$ \\
TNF- $\alpha$ (ng/g tissue) & $34.41 \pm 3.19$ & $35.89 \pm 2.9$ & $44.42 \pm 1.35^{* @}$ & $36.28 \pm 1.53^{\#}$ \\
\hline
\end{tabular}

Values are mean \pm S.E.M. $(n=7)$. ${ }^{*} P<0.05$, vs control; ${ }^{\#} p<0.05$, vs MTX rats; ${ }^{\circledR} p<0.05$, vs erdosteine-treated rats

\section{ERD protected hepatic architecture from MTX-induced toxicity}

Rats in control group had no lesions in architecture (Figure $1 \mathrm{~A}$ ), while the MTX-treated group showed cellular apoptosis represented by nuclear pyknosis, marked cytoplasmic eosinophilia and hepatic vacuolation, with disintegrated dark nuclei (Figures $1 \mathrm{C}$ and $1 \mathrm{D}$ ). In contrast, the MTX + ERD group had mild degree of hepatic degeneration, with improvements in histological architecture (Figure $1 \mathrm{E})$. The group that received only ERD treatment showed normal hepatic histology similar to that of the control group (Figure $1 \mathrm{~B}$ ).

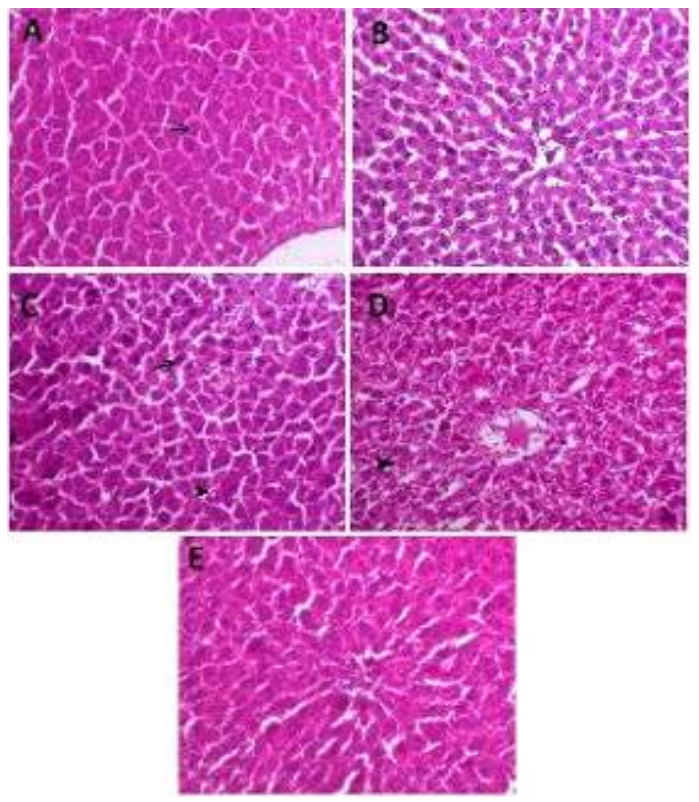

Figure 1: Erdosteine pre-treatment reduced methotrexate-mediated hepatic lesions in rats $(\mathrm{H} \& \mathrm{E}$, x200). A: Control liver section depicting normal liver cells radiating around the central vein (arrowed). B: $E R D$ liver section depicting normal liver architecture, with nucleus $(C, D)$. MTX group showing cellular apoptosis represented by nuclear pyknosis and increased cytoplasmic eosinophilia (arrowed) and hepatic vacuolation (arrowed). E: ERD + MTX group showing mild degree of hepatic degeneration and significant reduction in histopathological lesions

\section{ERD reduced apoptotic caspase-3 expression}

Rats in the control and ERD groups showed minimal caspase- 3 staining $(1.75 \pm 0.88,1.65 \pm$ 0.67 , respectively), whereas rats in the MTX toxicity group showed significantly higher caspase-3 staining $(20.5 \pm 2.20 ; p<0.05)$. These results are shown in Figure $2 \mathrm{~A}-\mathrm{C}$ and $\mathrm{E}$ ). In contrast, MTX + ERD rats had markedly reduced caspase-3 expression $(7.62 \pm 1.40)$, relative to the MTX toxicity rats $(p<0.05$; Figures $2 \mathrm{C}$ and D).

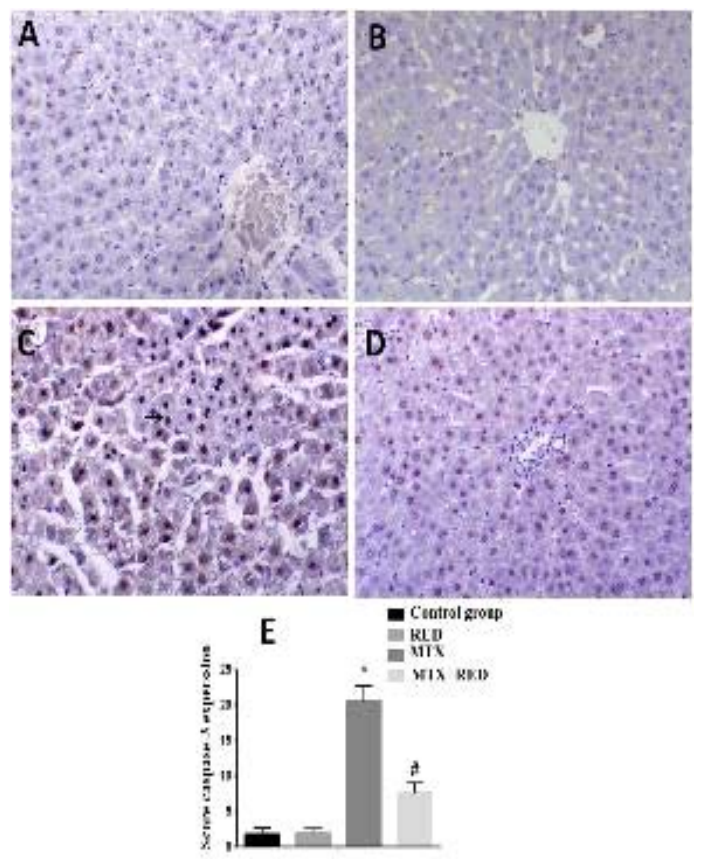

Figure 2: Erdosteine pretreatment reduced hepatic apoptotic marker (cleaved caspase 3 ) level in rats on methotrexate treatment. A: control group with low cleaved caspase 3 and cleaved caspase 3 IHC, x200. $B$ : ERD group showing no expression of cleaved caspase 3, cleaved caspase 3 IHC, X200. C: MTX group showing high nuclear expression of caspase 3 and cleaved caspase $3 \mathrm{IHC}, \mathrm{x200}$. D: ERD + MTX group showing marked decreases in caspase 3 expression and cleaved caspase 3 IHC, x200. E. Analysis of caspase-3 immuno-expression among different groups. Values represent mean $\pm S D(n=8)$ 


\section{DISCUSSION}

Hepatic toxicity due to MTX occurs through the inhibition of a number of anti-oxidant enzymes, leading to elevated oxidative stress and apoptosis. This investigation was carried out to study the potential mitigating effects of ERD against MTX-induced hepatotoxicity, based on the recently-recognized antioxidant properties of ERD. The findings showed significant increases in serum AST and ALT activities in MTX-treated rats, relative to control rats. It is known that AST and ALT are intracellular hepatic enzymes. Thus, their elevated serum levels are typical biochemical indicators of hepatocyte damage and loss of cell membrane integrity [16]. Previous studies in rat models have similarly reported elevated AST and ALT in association with MTX administration [17].

The basis of hepatotoxicity of MTX remains unclear. However, previous studies have suggested oxidative stress and the resultant apoptosis as key contributing factors in the process [5]. In this regard, increases in the concentration of the lipid peroxidation marker MDA in hepatic tissue specimens from MTXtreated rats, together with decreases in antioxidant enzyme activities of GSH, CAT, and SOD were observed, consistent with reports in previous studies [18]. Reactive oxygen species interact with cell membrane lipids resulting in the production of lipid peroxidation products which primarily affect the cell membrane and mitochondrial integrity, leading to cell death [19].

Moreover, various histopathological changes such as cellular apoptosis and hepatic vacuolation were observed in liver specimens from the MTX group. These changes were accompanied by increased expression of the proinflammatory cytokine TNF- $\alpha$ and the key cellular apoptotic protease caspase-3. Hepatic degenerative changes and apoptosis may be direct effects of MTX-induced inhibition of nucleic acid and protein synthesis, or an indirect outcome of mitochondrial energy depletion and oxidative stress [5]. With regard to inflammation, it has been established that despite the absence of microbial insults, inflammation can be triggered by apoptosis [20]. This occurs through the release of proinflammatory cell components from dead cells, or the release of proinflammatory cytokines from the hepatic phagocytes involved in removing the apoptotic bodies and cell debris [21]. Tumor necrosis factor-alpha (TNF- $\alpha$ ) is a potent proinflammatory cytokine known to trigger the recruitment of monocytes and neutrophils to enhance inflammation. Moreover, a synergistic interaction seems to occur between TNF- $\alpha$ and apoptosis.
Recent evidence suggests a role for TNF- $\alpha$ in the activation of caspase-dependent apoptotic pathways [22]. This may create a feedback loop of progressive inflammatory and apoptotic tissue damage.

The overall findings on changes in biochemical indicators of hepatic function and oxidative stress, in addition to the increased apoptotic marker caspase-3, and the observed histopathological alterations, are largely consistent with the pathological picture previously described for MTX-induced hepatotoxicity in rat models [9].

Interestingly, the pathological picture observed with MTX treatment was significantly suppressed with pre-conditioning treatment with ERD. The latter significantly reduced MTX-induced lipid peroxidation in liver tissue, as was evident in the significant decrease in MDA concentration and marked elevations in GSH, CAT and SOD in the liver, relative to MTX-treated rats. In addition, ERD ameliorated the apoptotic, inflammatory and degenerative changes associated with MTXtreatment, as indicated by reduced levels of TNF$\alpha$ and caspase-3.

The observed ERD-conferred protection can be attributed to its antioxidant activity. While ERD is well known as a mucolytic drug, its antioxidant properties have also been established over the last three decades [23]. The ERD molecule bears 2 atoms of sulfur, with one sulfur atom located in the aliphatic side chain, while the other is the thiolactone ring. After hepatic metabolism, ERD gives rise to various metabolites containing free carbon-bonded sulfhydryl groups (thiol groups). Thiol-containing metabolites, including ERD metabolites, are known for their intracellular antioxidant activities, either through direct scavenging of reactive oxygen species, or by replenishing glutathione stores [14].

Another possible mechanism that may explain the protective effect of ERD against MTXinduced hepatotoxicity is its demonstrated antiapoptotic effects. It has the potential to inhibit the proteolytic processing of caspase-3 and caspase-7, and the cytoplasmic release of the mitochondrial apoptotic factor cytochrome c, while enhancing the expression of bcl-2 and other survival proteins [24].

This study is the first to demonstrate the protective effect of ERD against hepatic toxicity associated with the use of MTX, and it adds to a vast body of evidence demonstrating its protective effect against drug-induced hepatotoxicity in general. It is noteworthy that the 
protective effects of ERD are likely mediated through its anti-oxidant effect, as previously demonstrated in organs other than the liver, e.g. heart [25].

\section{CONCLUSION}

The current study has demonstrated that erdosteine mitigates methotrexate-induced hepatotoxicity via its antioxidant and antiinflammatory effects. Moreover, it may have an anti-apoptotic effect.

\section{DECLARATIONS}

\section{Conflict of interest}

No conflict of interest is associated with this work.

\section{Contribution of authors}

We declare that this work was done by the authors named in this article and all liabilities pertaining to claims relating to the content of this article will be borne by the authors.

\section{Open Access}

This is an Open Access article that uses a funding model which does not charge readers or their institutions for access and distributed under the terms of the Creative Commons Attribution License (http://creativecommons.org/licenses/by/ 4.0) and the Budapest Open Access Initiative (http://www.budapestopenaccessinitiative.org/rea d), which permit unrestricted use, distribution, and reproduction in any medium, provided the original work is properly credited.

\section{REFERENCES}

1. Crawford S. Is it time for a new paradigm for systemic cancer treatment? Lessons from a century of cancer chemotherapy. Front Pharmacol 2013; 4: 1-18.

2. Czarnecka-Operacz M, Sadowska-Przytocka A. The possibilities and principles of methotrexate treatment of psoriasis - the updated knowledge. Postepy Dermatol Alergol 2014; 6: 392-400.

3. Rajagopalan PR, Zhang Z, McCourt L, Dwyer M, Benkovic SJ, Hammes GG. Interaction of dihydrofolate reductase with methotrexate: Ensemble and singlemolecule kinetics. Proc Natl Acad Sci U S A 2002; 99(21): 13481-13486.

4. Böhm I. Increased peripheral blood b-cells expressing the cd5 molecules in association to autoantibodies in patients with lupus erythematosus and evidence to selectively down-modulate them. Biomed Pharmacother 2004; 58(5): 338-343.

5. Bath RK, Brar NK, Forouhar FA, Wu GY. A review of methotrexate-associated hepatotoxicity. J Dig Dis 2014; 15(10): 517-524.

6. Bolser DC. Cough suppressant and pharmacologic protrusive therapy. Chest 2006; 129(1): 238S-249S.

7. Braga $P$, Sasso $M$, Zuccotti $T$. Assessment of the antioxidant activity of the metabolite of erdosteine on human neutrophil oxidative bursts. Arzneim-Forsch 2000; 50(08): 739-746.

8. Saritas $A$, Kandis $H$, Baltaci $D$, Yildirim U, Kaya $H$, Karakus A, Colakoglu S, Memisogullari R, Kara IH. Nacetyl cysteine and erdosteine treatment in acetaminophen-induced liver damage. Toxicol Ind Health 2014; 30(7): 670-678.

9. Akbulut S. Cytoprotective effects of amifostine, ascorbic acid and n-acetylcysteine against methotrexate-induced hepatotoxicity in rats. World J Gastroenterol 2014; 20(29): 10158-10165.

10. Kuvandik G, Duru M, Nacar A, Yonden Z, Helvaci R, Koc $A$, Kozlu $T$, Kaya $H$, Sogüt $S$. Effects of erdosteine on acetaminophen-induced hepatotoxicity in rats. Toxicol Pathol 2008; 36(5): 714-719.

11. Reitman S, Frankel S. A colorimetric method for the determination of serum glutamic oxalacetic and glutamic pyruvic transaminases. Am J Clin Pathol 1957; 28(1): 56-63.

12. Buege JA, Aust SD. Microsomal lipid peroxidation. Methods Enzymol 1978; 52: 302-310.

13. Ellman GL. Tissue sulfhydryl groups. Arch Biochem Biophys 1959; 82(1): 70-77.

14. Marklund S, Marklund G. Involvement of the superoxide anion radical in the autoxidation of pyrogallol and a convenient assay for superoxide dismutase. Eur $J$ Biochem 1974; 47(3): 469-474.

15. Aebi H. Catalase in vitro. Methods Enzymol 1984; 105 : 121-126.

16. Gowda S, Desai PB, Hull VV, Math AAK, Vernekar SN, Kulkarni SS. A review on laboratory liver function tests. Pan Afr Med J 2009; 3: 1-11. Biomed Pharmacother 2018; 97: 233-239.

17. Yoshida $Y$, Umeno A, Shichiri M. Lipid peroxidation biomarkers for evaluating oxidative stress and assessing antioxidant capacity in vivo. J Clin Biochem Nutr 2013; 52(1): 9-16.

18. Gaschler MM, Stockwell BR. Lipid peroxidation in cell death. Biochem Biophys Res Commun 2017; 482(3): 419-425.

19. Canbay A. Kupffer cell engulfment of apoptotic bodies stimulates death ligand and cytokine expression. Hepatology 2003; 38(5): 1188-1198.

20. Rock KL, Kono H. The inflammatory response to cell death. Annu Rev Pathol Mech Dis 2008; 3: 99-126.

21. Wang $L$, Du F, Wang $X$. Tnf- $\alpha$ induces two distinct caspase-8 activation pathways. Cell 2008; 133(4): 693703. 
22. Inglesi M, Nicola M, Fregnan G, Bradamante S, Pagani $G$. Synthesis and free radical scavenging properties of the enantiomers of erdosteine. Farmaco 1994; 40(11): 703-708.

23. Demiralay R, Gursan N, Erdem $H$. The effects of erdosteine and $n$-acetylcysteine on apoptotic and antiapoptotic markers in pulmonary epithelial cells in sepsis. Eurasian J Med 2013; 45(3): 167-175.
24. Yagmurca M, Bas O, Mollaoglu H, Sahin O, Nacar A, Karaman $O$, Songur A. Protective effects of erdosteine on doxorubicin-induced hepatotoxicity in rats. Arch Med Res 2007; 38(4): 380-385.

25. Selcoki Y, Uz E, Bayrak R, Sahin S, Kaya A, Uz B, Karanfil A, Ozkara A, Akcay A. The protective effect of erdosteine against cyclosporine a-induced cardiotoxicity in rats. Toxicology 2007; 239(1-2): 53-59. 